\title{
Sliding Mode Control Design for Hysteretic Ferroelectric Materials
}

\author{
Jerry A. McMahan and Ralph C. Smith \\ Department of Mathematics \\ Center for Research in Scientific Computation \\ North Carolina State University \\ Raleigh, NC 27695 \\ United States of America
}

\begin{abstract}
Ferroelectric materials are attractive for use in a wide range of applications due to their unique transduction capabilities. However, taking full advantage of these capabilities requires a control design which accounts for the materials' inherent hysteretic behavior. A common approach is to partially cancel the hysteretic effects in the system by employing an approximate inversion algorithm in the control input, resulting in an almost linear system. Using a recently developed modification to the homogenized energy model for ferroelectric materials, we combine this method with a sliding mode control design to track a reference trajectory even in the presence of modeling and inversion errors. Numerical simulations illustrate the effectiveness of the design.
\end{abstract}

Keywords: smart systems, hysteresis, sliding mode control, variable structure control, inverse control

\section{INTRODUCTION}

Ferroelectric materials possess attractive properties that make them well suited for many applications. Despite of advantages offered by ferroelectric actuators for emerging applications, such as low cost, light weight, high resolution and high bandwidth, the inherent hysteretic behavior they exhibit presents a challenge to utilizing these capabilities in high-performance designs. This has spurred significant research requiring the use of model-based control designs for these systems. A common approach is to apply inverse compensation methods to approximately cancel the hysteretic nonlinearities, resulting in an approximately linear system. ${ }^{1,2}$ Methods based on sliding mode control are also frequently employed ${ }^{3-7}$ (and not necessarily disjoint from inverse compensation methods), as they provide a means of accommodating the inevitable uncertainties that arise in modeling hysteresis.

In this work, we employ a control strategy incorporating both inverse compensation and sliding mode control. The methods are based on a recent extension to the homogenized energy model ${ }^{8}$ for ferroelectric materials. Our approach is similar to that of Ref. 9 which is concerned with tracking control of shape memory alloys, although we use a physics-oriented model and do not require an observer design for the measurement of our system state. The author of Ref. 10 also uses a similar approach to ours, although our design is based on classical sliding mode control, uses a different inverse, and a more recent material model.

The paper is organized as follows. In Section 2, we summarize the homogenized energy model quantifying the strain and polarization response to electric field and stress, as well as the actuator model incorporating the material physics and the hysteresis inversion algorithm employed to linearize the resulting system. In Section 3, we derive the sliding mode control law used for tracking a reference trajectory on the actuator system model. In Section 4, we describe the setup for the numerical simulation used to test the control, discusses implementation, and presents the simulation results. Finally in section 5, discusses the results and points to directions for future research.

Further author information:

J.A.M.: E-mail: jamcmaha@ncsu.edu

R.C.S.: E-mail: rsmith@eos.ncsu.edu, Telephone: 919-515-7552 


\section{MODEL DEVELOPMENT}

There are a number of models for hysteretic behavior in ferroelectric materials that are suitable for control design, each with various strengths and weaknesses. Phenomenological models are particularly popular for this purpose. These models are only concerned with accurately emulating the hysteretic response of a physical quantity to some physical input (e.g., output strain resulting from an input electric field) without quantifying the underlying physics responsible for the behavior. For example, the Preisach model describes a hysteretic curve by defining a family of elementary hysterons, each with different switching properties with respect to the input, and integrating them against a density function to compute the output. Another popular example is the Prandtl-Ishlinskii model, which has the key feature that an analytic inverse operator is known for certain operating conditions, ${ }^{11}$ avoiding the need for iterative techniques when compensating for the hysteresis. The lack of a physical basis for these types of models frees them from the additional constraints that more physically-oriented models may add, which accounts for their computational efficiency and suitability for implementation in real-time control applications. However this non-physical character obscures insight to the mechanisms responsible for the real-life behavior of the systems under consideration and may complicate incorporation of additional physical effects such as creep due to thermal relaxation, identification of model parameters, and rate-dependent hysteresis effects. ${ }^{12}$

The homogenized energy model (HEM) quantifies the hysteretic behavior in materials using a similar computational approach to the Preisach model, but uses a multiscale perspective to incorporate underlying physics. Hysterons describing the physical behavior at the lattice level are derived through energy minimization principles. The physical behavior at a macroscopic scale is considered as an aggregate of these smaller-scale relations and is calculated by integrating the elementary hysterons against a density. The result is a model with a computational structure and level of efficiency similar to the Preisach model, but with a clear physical interpretation for the mechanisms involved. This makes the incorporation of additional physical effects (like creep due to thermal relaxation and rate-dependent hysteresis) more natural. The added physical insight provides information which can be incorporated when developing practical techniques for parameter identification and control. Since variations of the model can describe the behavior of a number of materials exhibiting hysteretic behavior for different physical reasons (e.g., ferroelectrics, ferromagnetics, shape-memory alloys), comparison between these classes of materials is eased. This makes it easier to determine the applicability of engineering techniques across a broad range of materials.

We will use the homogenized energy model to derive the relation between the strain of a piezoelectric actuator and an input electric field. A simple approximate inversion algorithm will then be applied to linearize the resulting model. This linearized model will be the basis for our control design in the next section.

\subsection{Material Polarization and Strain}

Earlier work on the homogenized energy model for ferroelectric materials characterized the polarization response of a material to an applied electric field due to dipoles switching between domains oriented $180^{\circ}$ with respect to each other (i.e., dipoles switching from a positive-orientation to a negative-orientation and vice-versa). This material model was then incorporated into constitutive models describing the strain dynamics of piezoelectric actuators use in applications. Recent work ${ }^{8,13}$ incorporates the effects of $90^{\circ}$ switching to model both polarization and strain responses to applied electric fields and stresses. The consideration of $90^{\circ}$ switching in the model is important for accurately modeling strain, since $90^{\circ}$ switching is one of the primary strain-producing mechanisms in ferroelectrics. We will use the $90^{\circ}$ switching polarization and strain model as the basis for our model-based control design (although we will not need to use the polarization). In the following sections, we outline the equations which constitute the model. Derivation of the model is available in Ref. 8.

\subsubsection{Mesoscopic Scale Relations}

At the lattice level, dipoles are assumed to be restricted to three orientations: positively-oriented, negativelyoriented and $90^{\circ}$-oriented (respectively,,+- , and 90 in our notation). To quantify the switching, we define three variables, $x_{+}, x_{-}$, and $x_{90}$, which represent the fraction of dipoles of a given orientation in the reference volume being considered. For instance, $x_{+}=0.1$ signifies $10 \%$ of the dipoles are oriented positively. These variables are 
called the phase fractions. Assuming there are no instantaneous transitions directly between positively-oriented and negatively-oriented dipoles, the evolution equations for the phase fractions are

$$
\begin{aligned}
& \dot{x}_{+}=-\left(p_{90,+}+p_{+, 90}\right) x_{+}-p_{90,+} x_{-}+p_{90,+} \\
& \dot{x}_{-}=-p_{90,-} x_{+}-\left(p_{90,-}+p_{-, 90}\right) x_{-}+p_{90,-} \\
& x_{90}=1-x_{+}-x_{-}
\end{aligned}
$$

where the $p_{\alpha, \beta}$ coefficients are the transition rates from a $\alpha$-oriented dipole to a $\beta$-oriented dipole. The transition rates are defined as

$$
p_{\alpha, \beta}\left(E ; F_{c}\right)=\frac{1}{\tau_{\alpha, \beta}} \exp \left(-\gamma \Delta G_{\alpha, \beta}^{a}\left(E, \sigma ; F_{c}\right)\right)
$$

where $\tau_{\alpha, \beta}$ is the relaxation time of the material at the corresponding dipole orientation, $E$ is the applied electric field, $\sigma$ is the applied stress, $F_{c}$ is the thermodynamic driving force, and $\gamma=V / k T$ with $V$ the reference volume of the lattice, $T$ the temperature, and $k$ Boltzmann's constant. The $\Delta G_{\alpha, \beta}^{a}$ term in these equations is called the activation energy and is defined as

$$
\Delta G_{\alpha, \beta}^{a}\left(E, \sigma ; F_{c}\right)= \begin{cases}\left(1-F_{\alpha, \beta}(E, \sigma) / F_{c}\right)^{2} F_{c} / 4, & F_{\alpha, \beta}(E, \sigma) \leq\left|F_{c}\right| \\ F_{c}, & F_{\alpha, \beta}(E, \sigma)<-F_{c} \\ 0, & F_{\alpha, \beta}(E, \sigma)>F_{c} .\end{cases}
$$

The $F_{\alpha, \beta}(E, \sigma)$ term is a polynomial representing the thermodynamic force required to switch from an $\alpha$ orientation to a $\beta$ orientation, which is defined as

$$
F_{\alpha, \beta}(E, \sigma)=-\left(d_{\alpha}-d_{\beta}\right) E \sigma-\left(P_{R}^{\alpha}-P_{R}^{\beta}\right) E-\left(\varepsilon_{R}^{\alpha}-\varepsilon_{R}^{\beta}\right) \sigma
$$

where $d_{\alpha}$ is the piezoelectric constant (in units $\mathrm{C} / \mathrm{N}$ ), $P_{R}^{\alpha}$ is the remanence polarization, and $\varepsilon_{R}^{\alpha}$ is the remanence strain for the $\alpha$-oriented dipoles (and likewise for the $\beta$ superscript). These are material-dependent constants. While it is not our goal to discuss the derivation of these relations, to highlight the physical nature of these equations, we mention that $F_{\alpha, \beta}$ derives from subtracting the minimum of the Gibbs energy density of the $\beta$ orientation from that of the $\alpha$ orientation. The equations above are derived while considering thermal relaxation, so that creep effects are modeled as well.

The equations up to this point describe only the dynamics of the phase fractions. Polarization and strain are calculated at this domain level by using the values of the phase fractions as well as the applied electric field and applied stress to compute

$$
\begin{aligned}
\bar{d}\left(E, \sigma ; F_{c}\right) & =\sum_{\alpha=+,-, 90} d_{\alpha} x_{\alpha}\left(E ; F_{c}\right) \\
\bar{P}_{i r r}\left(E, \sigma ; F_{c}\right) & =\sum_{\alpha=+,-, 90} P_{R}^{\alpha} x_{\alpha}\left(E ; F_{c}\right) \\
\bar{\varepsilon}_{i r r}\left(E, \sigma ; F_{c}\right) & =\sum_{\alpha=+,-, 90} \varepsilon_{R}^{\alpha} x_{\alpha}\left(E ; F_{c}\right)
\end{aligned}
$$

where $\bar{d}$ is the piezoelectric constant, $\bar{P}_{i r r}$ is the irreversible polarization, and $\bar{\varepsilon}_{i r r}$ is the irreversible strain at the lattice level. These values are computed by considering the effects due to the different dipole orientations as material-dependent constants $\left(d_{\alpha}, P_{R}^{\alpha}\right.$, and $\left.\varepsilon_{R}^{\alpha}\right)$ and performing a weighted sum to determine the relative contribution of each orientation to the entire value. After these values are calculated, the polarization and strain at the lattice level are given by

$$
\begin{gathered}
\bar{P}(E, \sigma)=\bar{d}(E, \sigma) \sigma+\chi^{\sigma} E+\bar{P}_{i r r}(E, \sigma), \\
\bar{\varepsilon}(E, \sigma)=s^{E} \sigma+\bar{d}(E, \sigma) E+\bar{\varepsilon}_{i r r}(E, \sigma),
\end{gathered}
$$

where $\chi^{\sigma}$ is the ferroelectric susceptibility at constant stress and $s^{E}$ is the elastic compliance at constant electric field. This final calculation incorporates the linear $\left(\chi^{\sigma} E\right.$ and $\left.s^{E} \sigma\right)$, locally quadratic $(\bar{d}(E, \sigma) \sigma$ and $\bar{d}(E, \sigma) E)$, and irreversible contributions to to the total output. 


\subsubsection{Macroscopic Material Relations}

The lattice relations are adequate for modeling the behavior single-crystal of certain compounds in the absence of interaction fields. To characterize effects due to polycrystallinity, variable interaction fields, and material nonhomogeneities, the interaction field, $E_{I}$, and the thermodynamic driving force, $F_{C}$, are considered as manifestations of an underlying density. The expressions describing the lattice level behavior become a kernel representing the behavior over the range of values over which the density is defined, with the density assigning a weight to the contributions of these values. The kernel is integrated against this density to aggregate these contributions and obtain the macroscopic behavior using the equations

$$
\begin{gathered}
P(E, \sigma)=d(E, \sigma) \sigma+\chi^{\sigma} E+P_{i r r}(E, \sigma), \\
\varepsilon(E, \sigma)=s^{E} \sigma+d(E, \sigma) E+\varepsilon_{i r r}(E, \sigma),
\end{gathered}
$$

where

$$
\begin{gathered}
d(E, \sigma)=\int_{0}^{\infty} \int_{-\infty}^{\infty} \bar{d}\left(E+E_{I} ; F_{c}\right) \nu_{I}\left(E_{I}\right) \nu_{c}\left(E_{c}\right) d E_{I} d E_{c} \\
P_{i r r}(E, \sigma)=\int_{0}^{\infty} \int_{-\infty}^{\infty} \bar{P}_{i r r}\left(E+E_{I} ; F_{c}\right) \nu_{I}\left(E_{I}\right) \nu_{c}\left(E_{c}\right) d E_{I} d E_{c} \\
\varepsilon_{i r r}(E, \sigma)=\int_{0}^{\infty} \int_{-\infty}^{\infty} \bar{\varepsilon}_{i r r}\left(E+E_{I} ; F_{c}\right) \nu_{I}\left(E_{I}\right) \nu_{c}\left(E_{c}\right) d E_{I} d E_{c} .
\end{gathered}
$$

There are a number of possible choices for densities, but we choose a certain representation for its balance of accuracy, satisfaction of certain physically motivated constraints, and ease of computation. We use a weighted sum of lognormal densities for $F_{c}$ and of normal densities for $E_{I}$. The formulas for these are

$$
\begin{array}{rlrl}
\nu_{c}\left(F_{c}\right) & =c_{1} \sum_{k=k_{\alpha}}^{K_{\alpha}} \alpha_{k} \phi_{k}\left(F_{c}\right), & & \phi_{k}\left(F_{c}\right)=\frac{\exp \left(-\left[\ln \left(F_{c}\right)-\mu_{c}\right]^{2} / 2\left(\sigma_{c}^{k}\right)^{2}\right)}{\sigma_{c}^{k} F_{c} \sqrt{2 \pi}}, \\
\nu_{I}\left(E_{I}\right)=c_{2} \sum_{k=k_{\beta}}^{K_{\beta}} \beta_{k} \varphi_{k}\left(E_{I}\right), & \varphi_{k}\left(E_{I}\right)=\frac{\exp \left(-E_{I}^{2} / 2\left(\sigma_{I}^{k}\right)^{2}\right)}{\sigma_{I}^{k} \sqrt{2 \pi}},
\end{array}
$$

where $c_{1}, c_{2}$ are chosen to ensure integration to unity.

Numerical evaluation of the model uses standard numerical methods. Numerical quadratures are used to approximate the integrals, with the integration limits set to a finite value large enough to ensure sufficient accuracy. Numerical ODE solvers are used to compute the evolution of the phase fractions over the sample points determined from the quadrature rules. Efficient algorithms for computing the evolution of the phase fractions for the $180^{\circ}$ switching model are discussed in Ref. 14 , which are equally applicable to the $90^{\circ}$ model used in this work. A full discussion of the model implementation is available in. ${ }^{13}$

\subsection{Lumped Parameter Actuator Model}

A lumped parameter model of strain in a PZT actuator is derived in Ref. 8 by treating the actuator as a damped spring system and balancing forces. The input stress applied to the material is assumed constant, making the dynamics dependent on a single scalar input variable. The resulting model is

$$
\begin{aligned}
\dot{\varepsilon} & =-\frac{A+s^{E} L k}{s^{E} L c} \varepsilon+\frac{A}{s^{E} L c}\left(s^{E} \sigma_{0}+d\left(E, \sigma_{0}\right)+\varepsilon_{i r r}\left(E, \sigma_{0}\right)\right), \\
& =a \varepsilon+b \varepsilon_{m a t}(E),
\end{aligned}
$$

where $a=-\left(A+s^{E} L k\right) /\left(s^{E} L c\right), b=A /\left(s^{E} L c\right)$ are physical constants depending on the material and the geometry of the actuator, and $\varepsilon_{\text {mat }}(E)=s^{E} \sigma_{0}+d\left(E, \sigma_{0}\right)+\varepsilon_{i r r}\left(E, \sigma_{0}\right)$ is the strain in the rod material in response to at a constant prestress, $\sigma_{0}$, and a time-varying input field, $E$. The validity of this model relies on assumption that $-\sigma_{0}>L \varepsilon k / A+L \varepsilon c / A$ (note $\sigma_{0} \leq 0$ so $-\sigma_{0} \geq 0$ ). 


\subsection{Hysteresis Inversion Algorithm}

The model in (11) is nearly a linear system, save for the nonlinear coupling of the input, E. By treating $\varepsilon_{m a t}$ as the input to the system, techniques from linear control become applicable, as long as we have a method of determining a value of $E$ that produces a desired $\varepsilon_{m a t}$. This motivates the use of approximate hysteresis inversion methods. Representing the hysteresis inversion for our system as $\varepsilon_{m a t}^{-1}$, we can linearize the system to obtain the equation

$$
\begin{aligned}
\dot{\varepsilon} & =a \varepsilon+b \varepsilon_{\text {mat }}\left(\varepsilon_{\text {mat }}^{-1}(u)\right) \\
& =a \varepsilon+b u+b e
\end{aligned}
$$

where $e=b \varepsilon_{\text {mat }}\left(\varepsilon_{\text {mat }}^{-1}(u)\right)-b u$ is the inversion error. This simplifies our control design, as long as we are careful to choose methods which can accommodate the error term, be.

We use a simple, efficient method for inverting the hysteresis based on a binary search algorithm. The following steps describe the procedure:

1. Choose an input range for the input electric field over which the $\varepsilon_{\text {mat }}(E)$ is monotone (e.g., $0 \mathrm{MV} / \mathrm{m}$ to $2 \mathrm{MV} / \mathrm{m})$. Choose an error bound for $e$ that the control method can be reasonably expected to tolerate.

2. Choose a number, $N$, of points and divide the input range into an equally spaced number of $N$ points. In practice, certain choices of $N$ may be more suitable to the physical design of the system than others (e.g., if the input field is generated from an $m$-bit digital-to-analog converter, $N=2^{m}$ or $N=2^{k}$ for any $k \leq m$ would be sensible choices).

3. Input a desired value $\varepsilon_{m a t}^{d}$ and initialize $E$ to the middle point of the input range.

4. Compute $\varepsilon_{m a t}(E)$ and determine the difference from $\varepsilon_{m a t}^{d}$.

5. If the magnitude of the difference is smaller than the error tolerance chosen in the first step, then accept the current value of $E$ as the result and save the state of the phase fractions computed when calculating $\varepsilon_{\text {mat }}(E)$.

6. If the magnitude of the difference is larger than the error tolerance, then remove the lower half of the input range if the difference is negative or the upper half if the difference is positive. Set $E$ to the middle of the remaining range and repeat the process from step 4 until all input points are exhausted or the tolerance is met.

This algorithm inverts the hysteresis at a computational cost of no more than $O\left(\log _{2}(N-1)\right)$ times the computational cost of evaluating $\varepsilon_{\text {mat }}(E)$. $N$ need not be very high to obtain reasonable error tolerances. For instance, in the simulations described later, using $N=257$ resulted in a relative inversion error no worse than $3 \%$, even with some parameter error introduced to simulate imperfections in the parameter identification for the inverse model.

\section{SLIDING MODE CONTROL}

Sliding mode control is a control method which can achieve a control objective in the presence of uncertainties in the system provided some bounds on the uncertainty are known. To do this, a surface is defined on a system space which is typically a space of tracking errors in the system state with respect to some reference trajectory. The surface is defined so that trajectories which lie on it have some desired property. For example, in the space of tracking errors, the surface may be defined so that the system trajectory asymptotically approaches the reference trajectory. The control law is designed to force the trajectories in the system space to approach the surface and slide along it, so that the controlled system achieves the control objective. Since every point in the system spaces lies on one side of the surface or the other, a control structure that steers the system toward the surface is defined for each side. A state-feedback is used to determine which side of the surface the system trajectory is currently on and switch to the corresponding control structure. The process by which the control parameters 
are designed allows some uncertainty in the system to be accounted for while still enforcing convergence of the system trajectory to the surface.

We apply a sliding mode control to the system (12) with the objective of tracking a reference trajectory, following the procedure in Ref. 15. The inversion error, $e$, is treated as the system uncertainty which the control law will compensate for. In this work, we will only be simulating the system, so the source of uncertainty will be the error in the inversion algorithm and numerical errors due to finite accuracy in the simulation. For an experimental setup, modeling errors in the system would be incorporated as well, replacing some portion of the numerical uncertainty due to simulation.

\subsection{Sliding Mode Control Design}

The first step is to define the sliding mode surface that accomplishes our control objective when the system trajectory lies on it. For our purposes, we choose

$$
\begin{aligned}
s\left(\tilde{\varepsilon}_{1}, \tilde{\varepsilon}_{2}\right) & =\lambda \tilde{\varepsilon}_{1}(t)+\tilde{\varepsilon}_{2}(t), \\
\tilde{\varepsilon}_{2} & =\varepsilon-\varepsilon_{d}, \\
\tilde{\varepsilon}_{1} & =\int_{0}^{t} \tilde{\varepsilon}_{2}(\tau) d \tau,
\end{aligned}
$$

where $s$ is the sliding surface, $\lambda>0$ is a design parameter, and $\varepsilon_{d}$ is the desired or reference trajectory for the strain. Note that $\dot{\tilde{\varepsilon}}_{1}=\tilde{\varepsilon}_{2}$. When $s=0$, the error dynamics satisfy $\dot{\tilde{\varepsilon}}_{1}=-\lambda \tilde{\varepsilon}_{1}$, so that the tracking error will asymptotically approach zero. The choice of $\lambda$ affects how quickly the error converges to zero once the trajectory reaches the surface.

To ensure the sliding surface is eventually reached, the control is designed to satisfy the Lyapunov-like condition

$$
\frac{d}{d t} \frac{s^{2}}{2} \leq-\eta|s|
$$

where $\eta>0$ is a design parameter that determines the rate of convergence to the sliding surface. Evaluating this expression for our system, we get

$$
\frac{d}{d t} \frac{s^{2}}{2}=s \dot{s}=s\left(\lambda \tilde{\varepsilon}_{2}+a \varepsilon_{2}+b u+b e-\dot{\varepsilon}_{d}\right) .
$$

The first step in the control design is to solve for the control that ensures $s=0$ when there is no uncertainty in the system (i.e., when $e=0$ ). This ensures that trajectories lying on the surface stay on the surface when the system is perfectly characterized. For our system, such a control is

$$
u_{e q}=\frac{\dot{\varepsilon}_{d}-a \varepsilon_{2}-\lambda \tilde{\varepsilon}_{2}}{b}
$$

The next step is to add a term the control to ensure that (16) is satisfied even when uncertainties are present. We set

$$
u=u_{e q}-\frac{K \operatorname{sign}(s)}{b}
$$

and find an appropriate $K$. Replacing $u$ in (17) with this expression, the condition (16) becomes

$$
\begin{aligned}
\frac{d}{d t} \frac{s^{2}}{2} & =s\left(\lambda \tilde{\varepsilon}_{2}+a \varepsilon_{2}+b u_{e q}-K \operatorname{sign}(s)+b e-\dot{\varepsilon}_{d}\right) \\
& =s(b e-K \operatorname{sign}(s)) \leq-\eta|s|
\end{aligned}
$$


where the last step follows since $b$ is always a positive parameter. To satisfy this, $K$ must be chosen so that

$$
\begin{cases}K \geq \eta-b e, & s<0 \\ K \geq \eta+b e, & s>0\end{cases}
$$

for any possible value of the system error $e$. If a bound on the system error is known so that $|b e| \leq e_{\max }$, this is satisfied by choosing $K=\eta+e_{\max }$. Replacing $K$ by this expression, the control law becomes

$$
u=\frac{\dot{\varepsilon}_{d}-a \varepsilon_{2}-\lambda \tilde{\varepsilon}_{2}}{b}-\frac{\left(e_{\max }+\eta\right)}{b} \operatorname{sign}(s) .
$$

This is the value that is input to the hysteresis inversion algorithm to obtain the electric field value which is input to the system.

Inspection of the above steps may raise the question of what prevents the control from accommodating an unlimited amount of uncertainty. The answer lies in the discontinuous switching that occurs due to the $\operatorname{sign}(s)$ term. Larger uncertainty results in larger discontinuities when the sign of $s$ changes, which leads to more rapid changes in the system state. This results in the need for faster switching as the uncertainty rises. In physical systems, limitations in the input bandwidth place constraints on the magnitude of the uncertainty which can be tolerated. Detailed analysis of the trade-offs involved are available in Ref. 15 and Ref. 16.

\section{SIMULATION}

Numerical simulations were run to verify the expected behavior of the controlled system. An implicit Euler scheme was used to solve the ODE (12) with the control law (21) applied. We briefly discuss details of the implementation. A more thorough description of numerical methods for implementing the homogenized energy model is available in Ref. 13.

\subsection{System Setup}

The parameters for the system were taken from Ref. 8, which were obtained by a fit to experimental data from a PZT actuator. The reference trajectory chosen for the output strain was a $10 \mathrm{~Hz}$ sinusoid with a peak-to-peak amplitude of $0.02 \%$ at an offset of $0.03 \%$. This ensures the operation of the system in a hysteretic regime. The system was simulated at 5000 equally spaced points over a time interval of about 0.5 seconds, which corresponds to a time step size of $0.1 \mathrm{~ms}$.

A few implementation choices are worth noting as they have some impact on how well the results relate to practical implementation and on the uncertainty the control must account for.

1. The control input was updated every 10 time steps rather than every time step. As a practical system often has limits on how often the input can change, this allows for the robustness of the control with respect to these delays to be examined.

2. Since the control algorithm involves an approximate inversion of the hysteresis and this algorithm involves tracking the evolution of the phase fractions, the slower frequency of updating the control also exercises the robustness of the inversion algorithm. The higher errors in the estimation of the phase fraction leads to increased inversion error which the control must accommodate.

3. The input electric field was quantized to a set of 257 equally spaced values on the range $-2 \mathrm{MV} / \mathrm{m}$ to $2 \mathrm{MV} / \mathrm{m}$. While this is inherent to the hysteresis inversion algorithm used, this also corresponds to many practical systems where an input due to a control law will often be implemented digitally (albeit usually with some sort of post-filtering).

Two cases were simulated in order to test the performance of the control for different levels of system uncertainty.

1. The same parameters were used for the forward hysteresis model and the hysteresis inversion algorithm. 
2. In the hysteresis inversion algorithm, $5 \%$ error was introduced to the parameter values $\mu_{c}, \sigma_{c}$, and $\sigma_{I}$, which characterize the distributions for the model.

In the first case, sources of model uncertainty mostly come from quantization of the input in the inversion algorithm and the finite time update of the control input. The second case contains the same uncertainty effects as the first case, in addition to the effects of errors in estimating the material-dependent distribution parameters.

The control parameters were chosen through iterative simulation. For both test cases, $\lambda=10$ and $\eta=0.001$ were used. The uncertainty bound was different for each case, with $e_{\max }=0.56184$ used for the first case and $e_{\max }=1.6855$ used for the second. Note that $e_{\max }$ is $b$ times the maximum of the inversion error, $e$, from (12). For the parameters used in the system, this corresponds to a maximum inversion error magnitude (i.e., maximum value for $|e|)$ of $5 \times 10^{-6}$ for the first case and $1.5 \times 10^{-5}$ for the second. As seen in Figure 3 , these bounds are satisfied.

The values for $e_{\max }$ were chosen by simulating the system with some low guess for the value, calculating the bound on the resulting inversion error, and rerunning the simulation until the error did not exceed the new estimate. The initial conditions for the system were chosen to match the initial conditions for the reference trajectory so that the sliding surface could be reached in finite time (see the discussion in Ref. 15).

\subsection{Simulation Implementation}

To numerically evaluate the system (12), an implicit Euler scheme was used. For each time-step, this yields the implicit equation

$$
\varepsilon^{k+1}=\varepsilon^{k}+h\left(a \varepsilon^{k+1}+b \varepsilon_{m a t}\left(E^{k+1}\right)\right)
$$

where $h$ is the time step and the $k$ superscript implies the variable is evaluated at time $t=t_{0}+k h$. To solve this equation, a fixed point iteration on the system written in terms of $\varepsilon^{k+1}$. That is, (22) was solved for $\varepsilon^{k+1}$ to obtain

$$
\varepsilon^{k+1}=\frac{\varepsilon^{k}+h b \varepsilon_{m a t}\left(E^{k+1}\right)}{1-h a} .
$$

Note that this is still an implicit equation, since $E^{k+1}$ is the output of the hysteresis inversion algorithm with the control law (21) at time $t^{k+1}$. The control law at this time is dependent on $\varepsilon^{k+1}$ (among other variables) which means $E^{k+1}$ is as well. A fixed point iteration is used to solve this equation, using the previous state at $\varepsilon^{k}$ as the initial iterate.

The Matlab-compatible code used for the simulations is available for download at the link:

http://www4.ncsu.edu/ jhcrews/smart/code/pzt/index.html

\subsection{Results}

Figure 1 shows the results of the simulation for both test cases, comparing the outputs with the reference trajectories and showing the relative error. Both cases show reasonable tracking accuracy, with the second case showing a reduction in performance as expected due to its greater uncertainty. Figure 2 shows the input-output plots of the two test cases, confirming the system is operating in a hysteretic regime.

Figure 3 shows the error in the hysteresis inversion algorithm for each case. Again, uncertainty on the error is higher in the second case, but each case remains under the bound corresponding to the value chosen for $e_{\max }$. In the second test case, where there is additional parameter error in the inversion algorithm, the error slowly rises with time. This suggests further analysis on the effects of parameter errors on the inversion error may be worthwhile, to ensure the proper bounds on $e_{\max }$ are chosen.

Figure 4 shows the input electric field determined by the control algorithm for each case. There is not a significant amount of control chatter for these cases, although there is more for the second case than the first due to its greater uncertainty. A major reason for this is due to our simulation setup, where there is relatively little in the way of unmodeled dynamics, measurement error, etc., so it is significantly easier to get a tight bound on the uncertainty than in an experimental setup.

Figure 5 shows the value of equation for the sliding surface with the state trajectory as input. We see in both cases, the values are close to zero, with a higher deviation in the second case, as expected. 


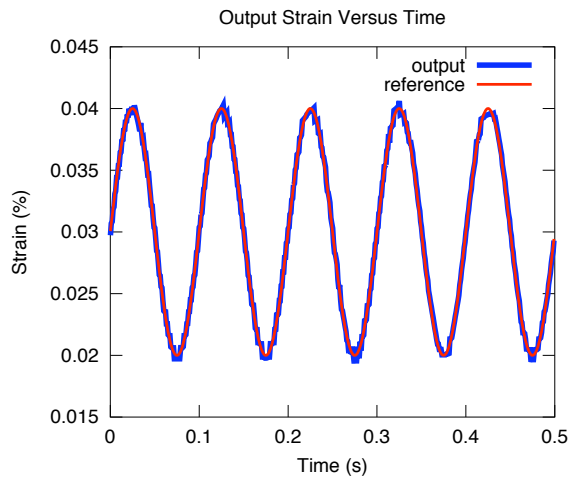

(a)

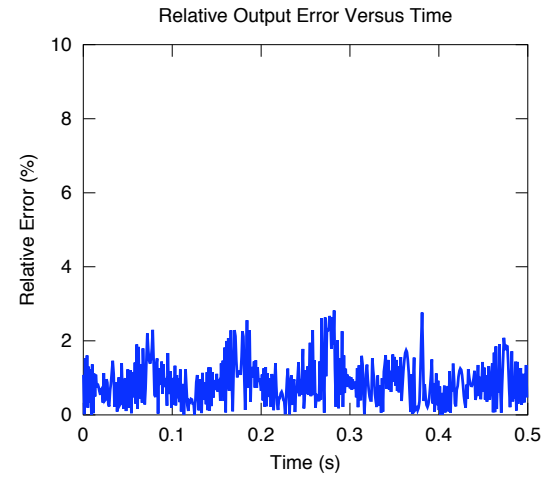

(c)

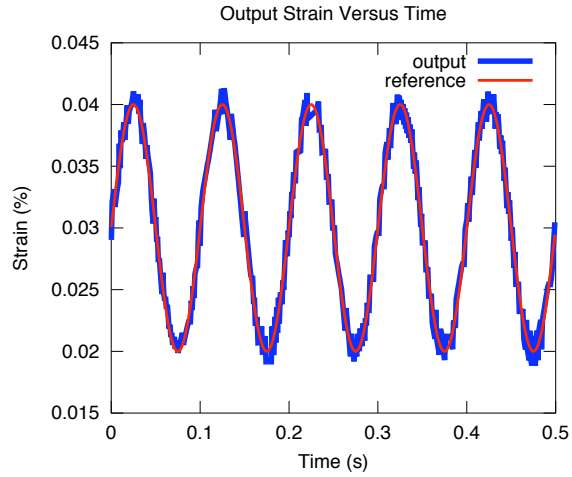

(b)

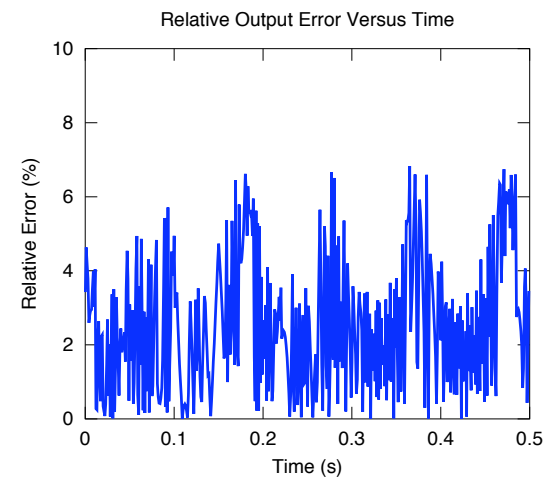

(c)

Figure 1. (a),(b): Output strain compared with reference strain. (c),(d): Relative output error. (a),(c): Test case 1. (b),(d): Test case 2 .

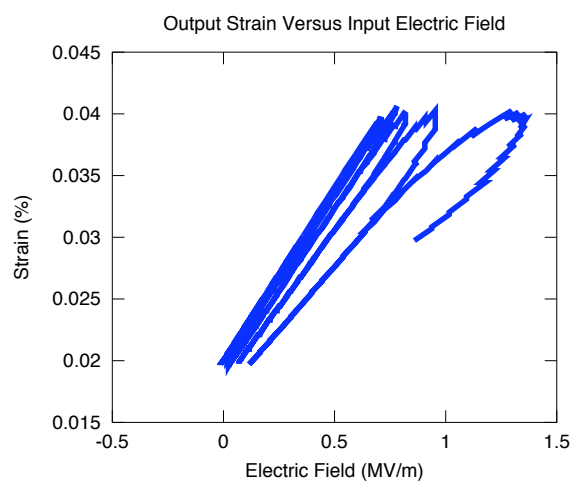

(a)

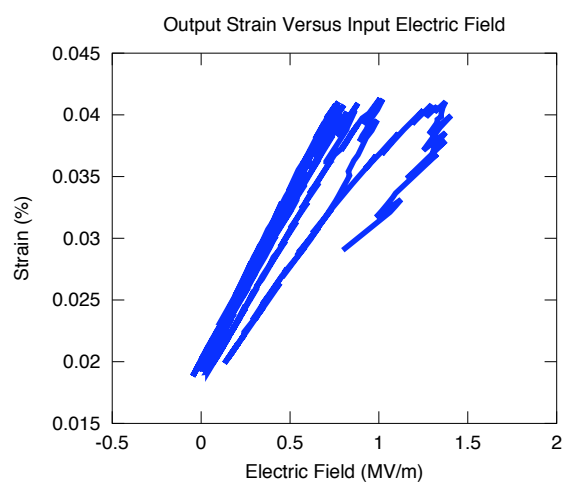

(b)

Figure 2. Hysteresis plots of output strain versus input for (a) test case 1 and (b) test case 2. 


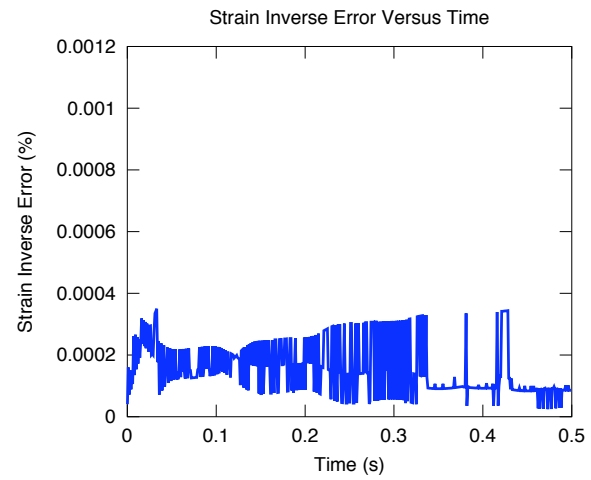

(a)

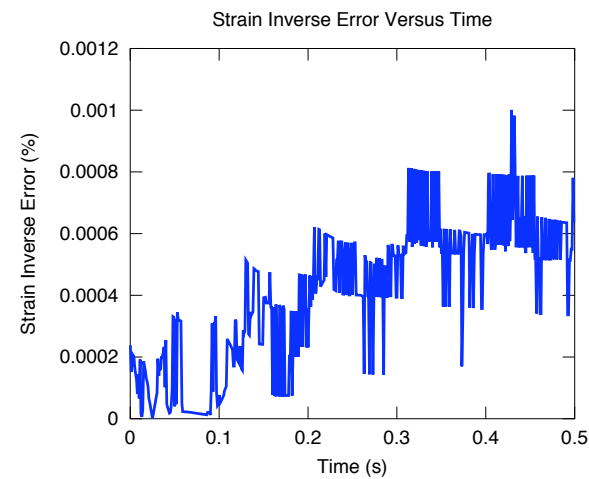

(b)

Figure 3. Inversion error for (a) test case 1 (b) test case 2.

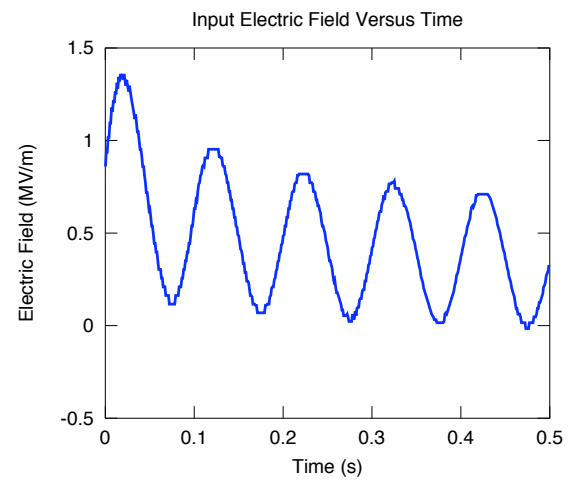

(a)

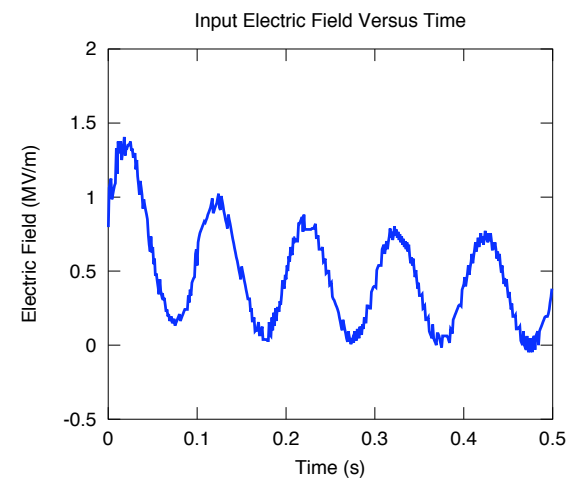

(b)

Figure 4. Input electric field for (a) test case 1 (b) test case 2.

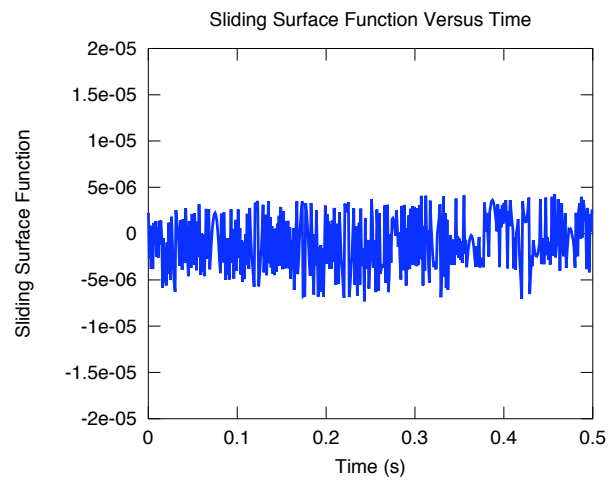

(a)

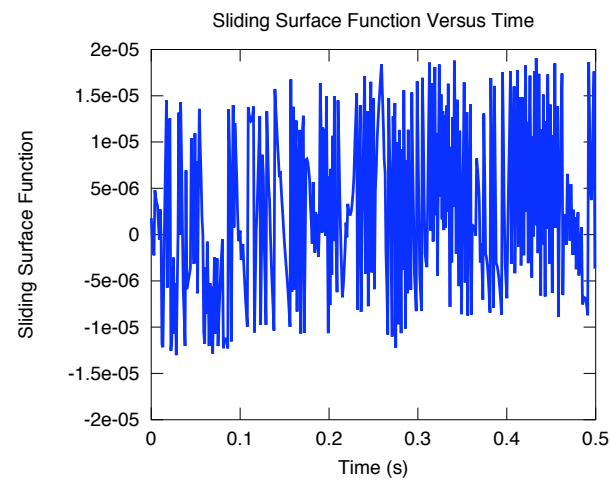

(b)

Figure 5. Sliding mode surface function evaluated for (a) test case 1 (b) test case 2 . 


\section{CONCLUSIONS AND FUTURE WORK}

The simulation results suggest that combining an approximate hysteresis inversion algorithm based on the homogenized energy model with a sliding mode control to compensate for the resulting uncertainty is a feasible approach to the tracking problem for ferroelectric actuators. However, the nature of the simulation limits the type of uncertainty which may be examined. An obvious step towards improving upon this is to test the controller in an experimental setup. In addition, it is not ideal to choose the control parameters through iterative testing, so additional analysis on the model parameters and their effects on the uncertainty would be beneficial. Incorporating adaptive techniques for determining the bounds on the model uncertainty would simplify the process of determining the control parameters, and provide additional robustness against transient uncertainties. Finally, as the testing apparatus is improved and the limitations of classical sliding mode control to the problem become more apparent, extensions to the control method, such as higher order sliding mode control or model predictive sliding mode control, may be tested.

\section{ACKNOWLEDGMENTS}

This research was supported in part by the Air Force Office of Scientific Research through the grant AFOSR FA9550-11-10152 and the NSF grant NSF DMS-0636590 EMSW21-RTG. We also thank John Crews for providing input regarding the paper.

\section{REFERENCES}

[1] Smith, R. C., Salapaka, M. V., Hatch, A., Smith, J., and De, T., "Model development and inverse compensator design for high speed nanopositioning," in [Proceedings of the 41st IEEE Conference on Decision and Control], 3652-3657 (December 2002).

[2] Krejci, P. and Kuhnen, K., "Inverse control of systems with hysteresis and creep," Control Theory and Applications, IEE Proceedings - 148, 185 -192 (May 2001).

[3] Xu, Q. and Li, Y., "Global sliding mode-based tracking control of a piezo-driven xy micropositioning stage with unmodeled hysteresis," in [The 2009 IEEE/RSJ International Conference on Intelligent Robots and Systems], 755-760 (October 2009).

[4] Shuanghe Yu, Bijan Shirinzadeh, G. A. and Smith, J., "Sliding mode control of a piezoelectric actuator with neural network compensating rate-dependent hysteresis," in [Proceedings of the 2005 IEEE International Conference on Robotics and Automation], 3641-3645 (April 2005).

[5] Chen, X. and Hisayama, T., "Adaptive sliding-mode position control for piezo-actuated stage," IEEE Transactions on Industrial Electronics 55(11), 3927-3934 (2008).

[6] Liaw, H. C., Shirinzadeh, B., and Smith, J., "Enhanced sliding mode motion tracking control of piezoelectric actuators," Sensors and Actuators A: Physical 138(1), $194-202$ (2007).

[7] Mohammad Sheikh Sofla, Seyed Mehdi Rezaei, M. Z. M. S., "Hysteresis-observer based robust tracking control of piezoelectric actuators," in [2010 American Control Conference], 4187-4192 (June 2010).

[8] Smith, R. C. and Hu, Z., "The homogenized energy model (HEM) for characterizing polarization and strains in hysteretic ferroelectric materials: Material properties and uniaxial model development," Preprint (2011).

[9] J. Hannen, J. C. and Buckner, G., "Indirect intelligent sliding mode control of a shape memory alloy actuated flexible beam using hysteretic recurrent neural networks," Submitted for review (December 2011).

[10] Kim, B., Design and Analysis of Model Based Nonlinear and Multi-Spectral Controllers with Focus on Motion Control of Continuous Smart Structures, $\mathrm{PhD}$ dissertation, Ohio State University, Columbus, $\mathrm{OH}$ (2010).

[11] Al Janaideh, M., Rakheja, S., and Su, C.-Y., "An analytical generalized prandtl-ishlinskii model inversion for hysteresis compensation in micropositioning control," Mechatronics, IEEE/ASME Transactions on 16, $734-744$ (Aug. 2011).

[12] Smith, R. C., [Smart Material Systems: Model Development], Frontiers in Applied Mathematics, Society for Industrial and Applied Mathematics, Philadelphia, PA (2005). 
[13] Smith, R. C. and Hu, Z., "The homogenized energy model (HEM) for characterizing polarization and strains in hysteretic ferroelectric materials: Implementation algorithms and data-model development and implementation," Preprint (2011).

[14] Braun, T. R. and Smith, R. C., "Efficient implementation algorithms for homogenized energy models," Continuum Mechanics and Thermodynamics 18, 137-155 (2006).

[15] Jean-Jacques E. Slotine, W. L., [Applied Nonlinear Control], Prentice Hall, Englewood Cliffs, NJ 07632 (1991).

[16] Utkin, V. I., [Sliding Modes in Control Optimization], Communications and Control Engineering Series, Springer-Verlag, Berlin Heidelberg New York (1992). 